\title{
Angioarchitecture of Spinal Dural Arteriovenous Fistula - Evaluation with 3D Rotational Angiography
}

\author{
Dae Chul Suh, MD1, Hye-Jin Baek, MD', Jee Won Park, MD', Ho Sung Kim, MD', \\ Kwang Kuk Kim, MD², Seung Chul Rhim, MD³
}

Purpose: The complex angioarchitecture of spinal dural arteriovenous fistulas (SDAVFs) sometimes preclude angiographic analyses or superselective procedures. Therefore, the effectiveness of 3 dimensional rotational angiography (3DRA) as a detailed imaging technique for SDAVFs was evaluated.

Materials and Methods: Of 57 patients with spinal vascular malformations, recent 13 SDAVF patients underwent 3DRA. The advantage of 3DRA compared to digital subtraction angiography (DSA) in imaging SDAVF was assessed. Angioarchitecture of SDAVF was focused on location, number, and course of feeders and draining vein. Appropriate angled views were also selected to reveal the segmental artery and feeders.

Results: 3DRA technique provided additional information for imaging evaluation of SDAVFs compared to DSA; the presence of multiple feeders, including their transdural portions, as well as their courses. The contralaterally angled anterior-oblique-caudal (spider) view showed the radicular feeder by separating the intercostal artery and the dorsal muscular branch. The bottom-to-up (tunnel) view was useful for revealing the location (ventral vs. dorsal) including sharp medial turn of the dural feeder. The dual mode, which displays both vessels and bones, revealed the course of the feeders and the fistula related to the spinal bony column.

Conclusion: Because spinal vasculature overlaps in DSA, 3DRA revealed additional information for evaluations of the number and transdural course of fistular feeders in SDAVFs, and it offers working angles to obtain appropriate views.

Key Words : Arteriovenous fistula; Spine; Imaging, Three-dimensional

This study was supported by a grant from the Korea Healthcare Technology R \& D Project, Ministry of Health \& Welfare, Republic of Korea (A080201).

Departments of ${ }^{1}$ Radiology and Research Institute of Radiology, ${ }^{2}$ Department of Neurology, ${ }^{3}$ Department of Neurological Surgery, University of Ulsan, College of Medicine, Asan Medical Center, Seoul, Korea

Received July 15, 2011; accepted after revision September 25, 2011.

Correspondence to: Dae Chul Suh, MD, Department of Radiology, Asan Medical Center, University of Ulsan, College of Medicine, 86 Asanbyeongwon-gil, Songpa-gu, Seoul 138-736, Korea.

Tel. 82.2.3010.4366 Fax.82.2.476.0090 E-mail: dcsuh@amc.seoul.kr

This is an Open Access article distributed under the terms of the Creative Commons Attribution Non-Commercial License (http://creativecommons.org/licenses/by-nc/3.0) which permits unrestricted non-commercial use, distribution, and reproduction in any medium, provided the original work is properly cited. 
Spinal dural arteriovenous fistulas (SDAVFs) are the most common form of spinal vascular disease, occurring in about $70 \%$ of spinal vascular malformation cases [1,2] and $56 \%$ of our patient series [3]. Despite being the most commonly encountered spinal vascular malformation, SDAVFs are rare and still underdiagnosed entities, which, if not treated properly, can lead to considerable morbidity with progressive spinal cord symptoms $[2,4]$. Venous congestive myelopathy (VCM) is a characteristic feature of SDAVF patients and is caused by the regurgitation of fistular flow through the radicular vein to the perimedullary venous plexus $[5,6]$.

Several case studies of three-dimensional rotational angiography (3DRA) applied to the spinal cord have provided useful 3D information about spinal angioanatomy and the locations of vessels relative to the spinal cord and surrounding structures because spinal 3DRA images provided better understanding of vessel architecture and vascular lesions in spinal arteriovenous malformations (AVMs) than two-dimensional (2D) angiography [7-9].

Revealing the angioarchitecture of SDAVFs is not easy because the fistula does not have a high flow or a large lumen size of feeders. In addition, the feeder is overlapped on 2D angiography with numerous segmental arterial branches precluding identification of
SDAVFs. Therefore, we assessed the advantages of 3DRA in imaging the angioarchitecture of SDAVFs and described a technical protocol and analyses to maximize these advantages.

\section{MATERIALS AND METHODS}

Among 57 patients with spinal vascular malformations that were documented in a neurointerventional database that contained data that were prospectively collected since 1992, recent 16 patients underwent three-dimensional digital subtraction rotational angiography using the Axiom Artis dBA system (Siemens Medical Solutions, Forchheim, Germany). In these patients, there were 13 SDAVFs and three spinal pial arteriovenus fistulas (SPAVFs). SDAVFs were the most common form of spinal vascular disease $(56 \%)$ at our institute. Thirteen SDAVF patients (age range: $34-77$, mean: $57 ; \mathrm{M}: \mathrm{F}=11: 2$ ) were out of 32 SDAVF which were the most common form of spinal vascular disease $(56 \%)$ at our institute.

Lesions were found at the cervical $(\mathrm{n}=1)$, thoracic ( $\mathrm{n}$ $=9)$ and lumbosacral $(\mathrm{n}=3)$ levels. Spinal MRI revealed typical VCMs in all SDAVF patients as we described previously [3]. Nine patients with SDAVFs underwent embolisation and two underwent surgical resection. One SDAVF patient refused treatment. Data

Table 1. Summary of Angiographic Findings from 13 SDAVF patients

\begin{tabular}{|c|c|c|c|c|c|c|c|c|}
\hline \multirow[t]{2}{*}{ No. } & \multirow[t]{2}{*}{ Age } & \multirow[t]{2}{*}{ Sex } & \multirow[t]{2}{*}{ Lesion level } & \multirow[t]{2}{*}{ No. of feeders } & \multirow[t]{2}{*}{ Feeder course } & \multicolumn{2}{|c|}{ Course of draining vein } & \multirow[t]{2}{*}{$\mathrm{Tx}$} \\
\hline & & & & & & Radicular vein & Perimedullary vein & \\
\hline 1 & 45 & M & $C 4-5$ & 1 & $\uparrow$ & $\uparrow$ & $\uparrow$ & $\mathrm{Em}$ \\
\hline 2 & 67 & $\mathrm{M}$ & T5 & Plexiform & $\downarrow$ & $\uparrow$ & $\uparrow$ & $\mathrm{Em}$ \\
\hline 3 & 57 & M & T5 & 1 & $\downarrow$ & $\uparrow$ & $\uparrow$ & ND \\
\hline 4 & 56 & M & T6 & 1 & $\uparrow$ & $\uparrow$ & $\downarrow$ & $\mathrm{Em}$ \\
\hline 5 & 46 & M & T6 & 2 & $\downarrow$ & $\uparrow$ & - & $\mathrm{Em}$ \\
\hline 6 & 49 & $M$ & T7 & 2 & $\rightarrow$ & $\uparrow$ & $\downarrow$ & Op \\
\hline 7 & 77 & $\mathrm{M}$ & T10 & 2 & $\rightarrow$ & $\uparrow$ & $\downarrow$ & Op \\
\hline 8 & 69 & $\mathrm{~F}$ & T10 & 2 & $\rightarrow$ & $\uparrow$ & - & $\mathrm{Em}$ \\
\hline 9 & 34 & M & $\mathrm{T} 11$ & 1 & $\downarrow$ & $\uparrow$ & $\uparrow$ & $\mathrm{Em}$ \\
\hline 10 & 52 & M & T11 & 1 & $\uparrow$ & $\uparrow$ & $\uparrow$ & $\mathrm{Em}$ \\
\hline 11 & 63 & $\mathrm{~F}$ & L3 & 2 & $\uparrow$ & $\uparrow$ & $\uparrow$ & $\mathrm{Em}$ \\
\hline 12 & 69 & $M$ & L3 & 1 & $\rightarrow$ & $\uparrow$ & - & $\mathrm{Em}$ \\
\hline 13 & 63 & M & S1 & Plexiform & $\rightarrow$ & $\downarrow$ & $\uparrow$ & $\mathrm{Em}$ \\
\hline
\end{tabular}

Signs: $\uparrow=$ Ascending; $\downarrow$ = descending; $\rightarrow=$ transverse; $\uparrow$ = bidirectional courses

Abbreviations: $\mathrm{M}$, male; Tx, treatment; $\mathrm{Em}$ = embolization; $\mathrm{ND}=$ not done; $\mathrm{Op}=$ operation; $\mathrm{F}$, female 


\section{Dae Chul Suh, et al.}

collection and analysis for this prospective $3 \mathrm{D}$ angiography study of spinal AVMs were performed according to our local institutional review board guidelines, and written informed consent was obtained from all patients and their families.

\section{DRA protocol}

The rotational angle was $200^{\circ}$ and the rotational speed of the $\mathrm{C}$-arm was $45^{\circ} /$ second. Data were acquired in a $2480 \times 1920$ matrix using a 16 -inch field-of-view flat panel detector. Two-hundred and seventy $\mathrm{mgI} / \mathrm{ml}$ of nonionic contrast medium (Visipaque, GE Healthcare, Chalfont, St. Giles, United Kingdom) was injected into segmental arteries (flow rate: $1 \mathrm{~mL} / \mathrm{s}$; total volume: $5 \mathrm{~mL}$ ), and the injection started 1 second before the rotational run to achieve complete filling of the selected artery during angiography. Both mask data and contrast data were transferred to a workstation (Syngo X-Workplace, Siemens AG, Healthcare Sector, Erlangen, Germany) and reconstructed into 3D images using a maximal intensity projection reconstruction algorithm.

Reconstructed images, including maximum intensity projection images, shaded surface-rendered displays, and volume-rendered displays with adjustable transparencies for various structures, in addition to full stereoscopic capabilities, were used to study the anatomic relationships shown in these images.

\section{Analysis of 3D angiograms}

To assess the additional information obtained by 3DRA, two neuroradiologists (H.J.B., D.C.S.) evaluated pretherapeutic 3DRA reconstructed images compared to $2 \mathrm{D}$ angiograms with respect to three categories of parameters graded by four scores $(1=$ poor, 2 = fair, 3 = good, $4=$ excellent). The three categories were: (1) the exact anatomic location of the fistula; (2) the precise angioarchitecture, including the number and course of feeding arteries and draining veins; and (3) the contribution of 3DRA to additional interventions that facilitated treatment, modified therapeutic strategies or improved safety.

Although most SDAVFs consisted of a fistula at a single segmental artery level, multiple channels of feeders at each level of the fistula were sometimes observed. The number and course of feeders and draining radicular veins were evaluated because the angioarchitecture, including the course of feeders while traversing the neural foramen, is critical for embolisation and surgical resection. Although radicular branches usually go upward along the radicular root below the pedicle, the feeder arising from the radicular artery or arising separately from the radicular artery took diverse courses in SDAVFs. Therefore, directions were categorised as upward, horizontal or downward and demarcated on the dual volume window, which shows vessels and bone (Fig. 1).

After obtaining the 3D angiography dataset, the most appropriate image was chosen by controlling the image window and the contrast on the workstation, and 3D images were reviewed at different angles. The most suitable views were chosen to reveal the two most complex areas of the feeder angioarchitecture: (1) the level where the segmental artery branches into intercostal, radicular and dorsal muscular arteries, and (2) the level where the radicular artery makes a sharp medial turn into the intervertebral foramen.

Inter-observer agreement on the angiographic findings was assessed, and analyses of the number of feeders and the course of feeders were performed by two neuroradiologists (H.J.B., D.C.S.). Data were analysed using Cohen's kappa statistics, and calculations were performed with MedCalc (MedCalc Software version 9.0, Mariakerke, Belgium).

\section{RESULTS}

\section{Analysis of 3D angiograms}

The angioarchitectures of 13 SDAVF patients are summarized in Table 1. Although the radicular arterial feeder and fistula were expected to have an oblique ascending course, the feeder courses at the neural foramen were diverse: horizontal $(\mathrm{n}=5)$, oblique upward $(n=4)$, and oblique downward $(n=4)$ (Fig. 1). Such diverse courses of the feeder from the radicular artery were characterised by a variable length of the transdural portion of the fistula before draining into the radicular vein, which depended on the anatomical position of the neural foramen.

The number of feeders at the lesion level was one (n $=6)$, two $(n=5)($ Figs. 1, 2), or plexiform $(n=2)$. The dual mode (showing bone and vessels) of 3D images clearly demonstrated such feeder courses relative to the vertebral pedicle (Fig. 1). The courses of veins at the level of the fistula showed ascending $(n=10)$, descending $(n=1)$ or bidirectional $(n=2)$ patterns. Draining veins with an ascending pattern regurgitated to perimedullary veins at the level of the spinal cord bidirectionally $(n=6)$, only downward $(n=3)$ or upward $(n=1)$. Perimedullary venous drainage was not identified in three patients. Both readers gave high scores (mean 3.9), reflecting the overall agreement 

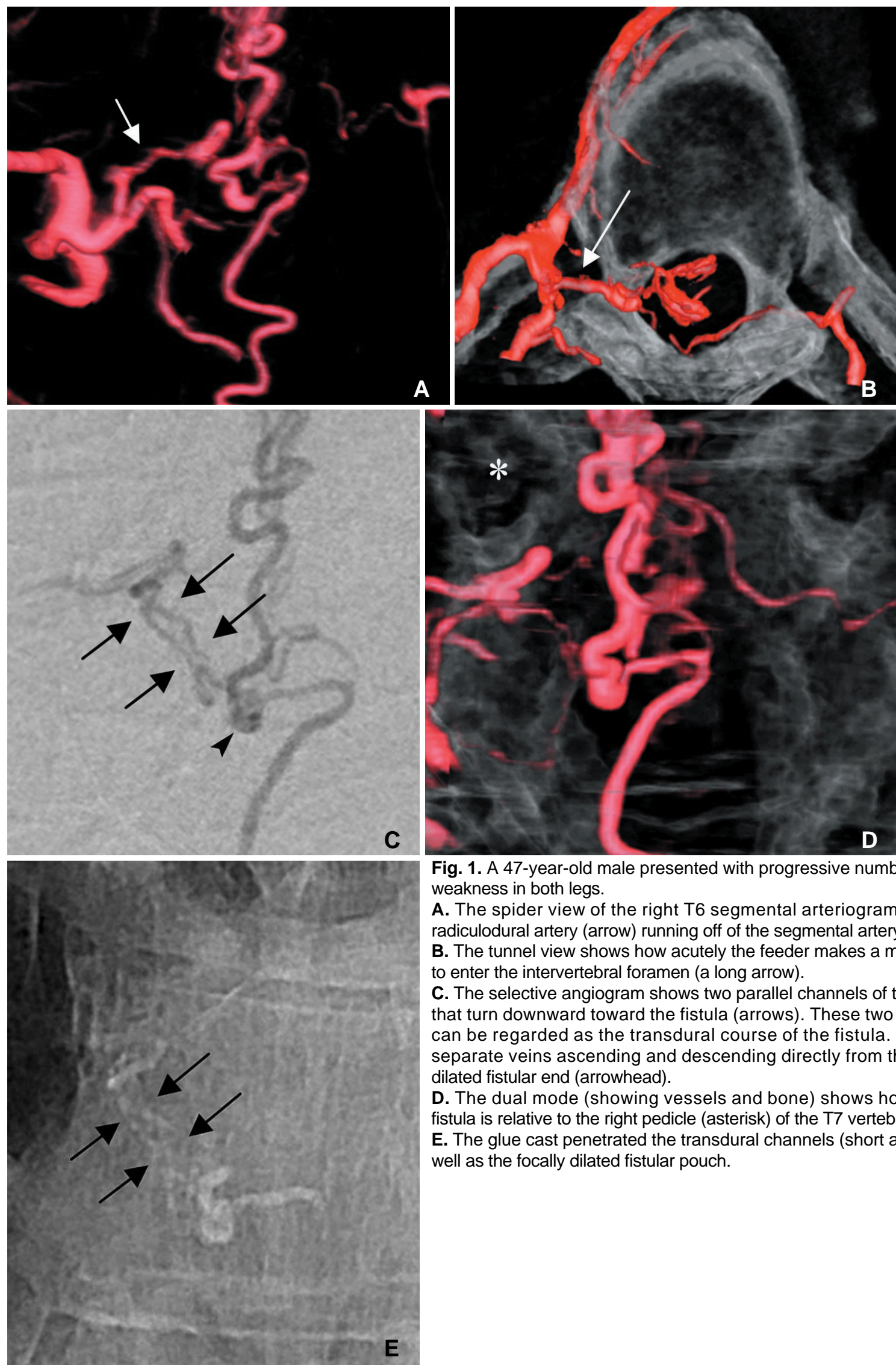

Fig. 1. A 47-year-old male presented with progressive numbness and weakness in both legs.

A. The spider view of the right T6 segmental arteriogram shows a radiculodural artery (arrow) running off of the segmental artery.

B. The tunnel view shows how acutely the feeder makes a medial turn to enter the intervertebral foramen (a long arrow).

C. The selective angiogram shows two parallel channels of the feeder that turn downward toward the fistula (arrows). These two channels can be regarded as the transdural course of the fistula. Note two separate veins ascending and descending directly from the focally dilated fistular end (arrowhead).

D. The dual mode (showing vessels and bone) shows how far the fistula is relative to the right pedicle (asterisk) of the T7 vertebra.

E. The glue cast penetrated the transdural channels (short arrows) as well as the focally dilated fistular pouch. 
Dae Chul Suh, et al.

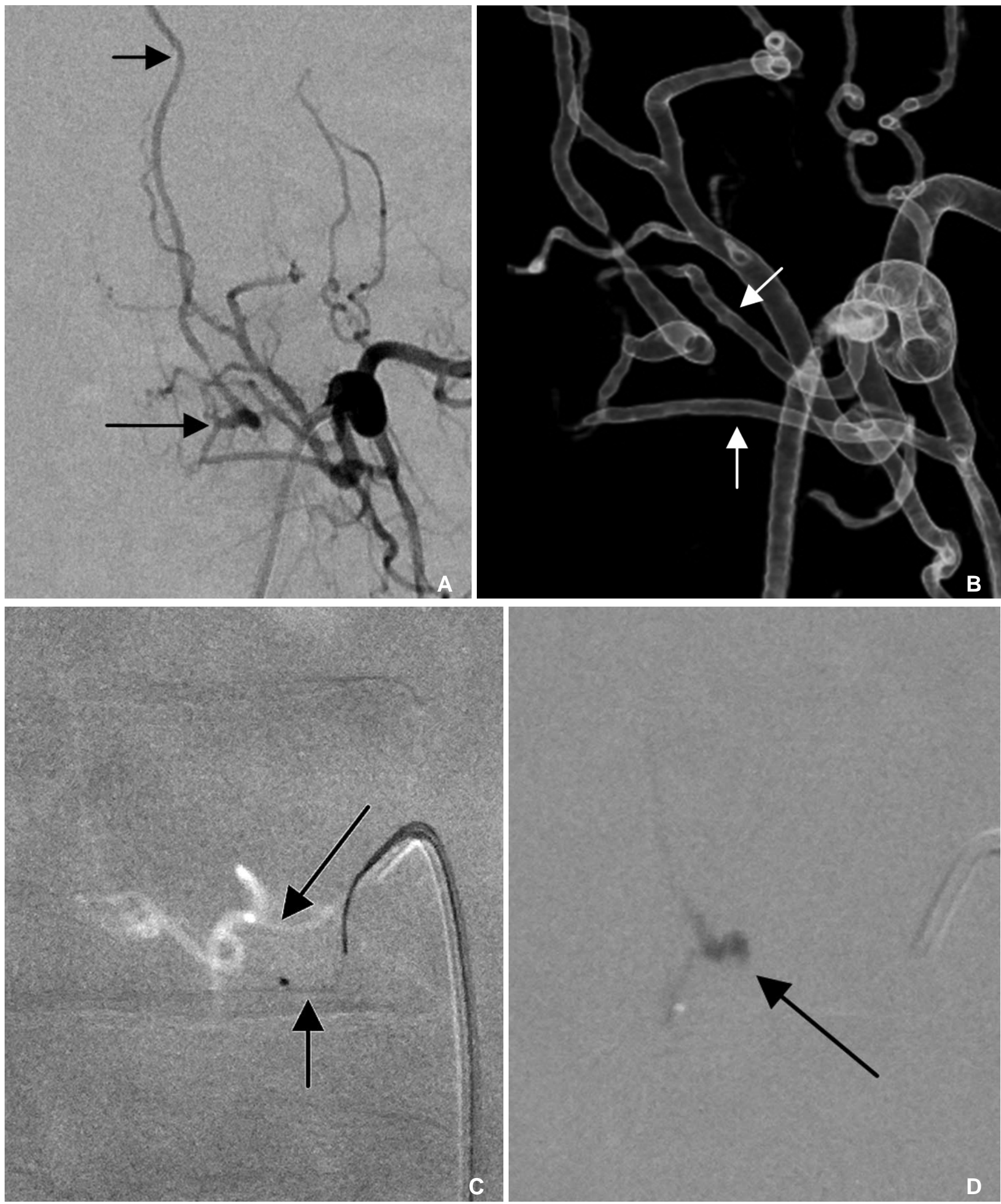

Fig. 2. A 64-year-old female presented with voiding difficulty and weakness in both legs for two months.

A. The left L3 lumbar arteriogram shows a SDAVF (arrow) draining into the ascending radicular vein (short arrow).

B. The 3D angiogram reveals two separate feeders (arrows) converging onto a fistula.

C. The lower feeder (a short arrow) was selected because the upper one (a long arrow) was difficult to select due to the acute angle of its origin.

D. A small amount of glue cast penetrated the proximal radicular vein (arrow), occluding the view of the other feeder. 
with excellent presence of additional information obtained by 3DRA. Inter-observer evaluations showed good agreement for the number of feeders $(\kappa=0.769)$, the course of feeders $(\kappa=0.893)$ and the course of veins $(\kappa=0.809)$.

\section{Selection of spider and tunnel views}

Two useful views were used to follow the proximal branching pattern of each segmental artery and the distal branching pattern of the radicular artery entering the transverse foramen. The first one (spider view) isolated the feeder by showing the branching pattern of the segmental artery (Fig. 1). The angles were the right anterior oblique (RAO) view of the feeder's right side and the left anterior oblique (LAO) view of the feeder's left side. The second one (tunnel view) showed the feeder course at the neural foramen and the location (ventral vs. dorsal) including sharp medial turn of the feeder.

Spider and tunnel views provided better working angles on 3D workstations, especially during embolisation. Different angles of the spider view were selected for each side. The best working angle was obtained at $\mathrm{RAO} / \mathrm{LAO} 30^{\circ}$ and caudal $20^{\circ}$. This angle was selected through the synchronised rotation of the $3 \mathrm{D}$ image on the console during embolisation, and it depended on which vessel segment was analysed and how much the microcatheter advanced. The dual mode showing bone and vessels revealed the locations of feeders and their courses.

\section{DISCUSSION}

Our study provides additional evidence that spinal 3DRA is useful for evaluations of the angioarchitecture of SDAVFs including the course of feeders. We also recommend useful and routine angled (spider and tunnel) views for 3D angiographic analysis. These routine angled views allow for the careful scrutiny of branch details. The spider view (contralateral-obliqueangled caudal view) and the tunnel view (bottom-up view) are useful for distinguishing the proximal branching vessels of the intercostal artery, the dorsal muscular arteries, and the fistula from the radicular artery with its sharp medial turn from the segmental artery. The dual mode of spinal 3DRA reveals the relationship between the fistula and bony landmarks, so these images provide a good roadmap for feeder selection during embolisation as well as for surgical guidance without obtaining further conbeam CT angiography [10]. 3DRA with the routine views (spider and tunnel views) proposed in our study allows for the easy selection of working angles and separates the complex and overlapping vascular anatomy of feeders.

The radicular artery, which branches from the segmental artery, is known to be associated with the main feeder in SDAVFs, and it usually has an obliquely ascending course because the fistula is located in the dura adjacent to the nerve root [11]. However, only one-third of our patients had feeders with an upward oblique course, and the majority of patients had feeders with a transverse or downward course that possibly traveled along the dura between two adjacent nerve roots (intersegmental) [11]. This discrepancy may originate from the different anatomical positions of the radicular vein and artery; in contrast to the radicular artery, the radicular vein does not follow the segmental rules [12]. The radicular vein pierces the dura and nerve roots in only $60 \%$ of cases; in the remaining $40 \%$, there is a separate foramen in the dura, between the two spinal nerves. Such orientations shown in the dual mode, which reveals bone and vessels, are helpful for embolisation as well as surgical planning.

SDAVFs are known to have a single feeder. However, the exact angioarchitecture of SDAVFs at the lesion level has not been well described. We observed that SDAVFs consisted of multiple feeders in 54\% of patients. The feeders even formed plexiform channels in which each feeder was not separately identified in two patients. Multiple feeders result in dual arterial supply to SDAVFs, which makes it imperative at the time of treatment to reach the venous outlet of the shunt, because otherwise the shunt will continue to be fed by the untreated arterial pedicle [11]. On the other hand, reciprocal communication in each feeder can cause glue injection at the wedged position of one feeder to regurgitate in a retrograde fashion, while making the glue penetrate the fistula and the proximal radicular vein. If there is any intersegmental anastomosis connecting to the anterior spinal artery in the adjacent level, such proximal regurgitation can lead to serious complications caused by glue inadvertently entering the anterior spinal artery [13]. Therefore, 3DRA can be helpful for evaluating the anatomy of multiple feeders and provide a good roadmap for further superselection and treatment of the fistula (Fig. 1).

Our study has several limitations. First, the number of patients is small because SDAVFs are a relatively rare occurrence. Second, obtaining 3DRA requires complex and high-quality angiographic equipment with threedimensional and rotational capabilities. Using 3DRA may increase the cost, radiation exposure and duration 


\section{Dae Chul Suh, et al.}

of the procedure. Third, the selection of the segmental artery from the aorta and the stable engagement of the catheter to perform 3DRA are not always easy because of the relatively acute angle of the segmental artery run-off from the aorta. Differently shaped catheters might be required for different levels of the segmental artery.

In conclusion, 3DRA provided additional information for evaluating the angioarchithecture of SDAVFs including the number and course of feeders, and for choosing appropriate working angles to select feeders and achieve successful embolisation or surgical treatment.

\section{Acknowledgments}

We acknowledge the assistance of Sun Moon Whang, B.S., Ok Kyun Lim, B.S., Eun Hye Kim, R.N. in collecting patient data, as well as that of Yun Gyeong Jeong in preparing the manuscript.

\section{References}

1. Thron A, Caplan LR. Vascular malformations and interventional neuroradiology of the spinal cord. In: Brandt T, Caplan LR, Dichgans J, Diener HC, Kennard C. Neurological disorders: course and treatment. Amsterdam: Academic Press, 2003:517-528

2. Krings T, Geibprasert S. Spinal dural arteriovenous fistulas. $A J N R$ Am J Neuroradiol 2009;30:639-648

3. Lee C-S, Pyun HW, Chae EY, Kim K-K, Rhim SC, Suh DC. Reversible aggravation of neurological deficits after steroid medication in patients with venous congestive myelopathy caused by spinal arteriovenous malformation. Interv Neuroradiol 2009;15:325-329

4. Jellema K, Tijssen CC, Sluzewski M, van Asbeck FW, Koudstaal PJ, van Gijn J. Spinal dural arteriovenous fistulas - an underdiagnosed disease. A review of patients admitted to the spinal unit of a rehabilitation center. $J$ Neurol 2006;253:159-162

5. Suh DC, Choi CG, Sung KB, Kim KK, Rhim SC. Spinal osseous epidural arteriovenous fistula with multiple small arterial feeders converging to a round fistular nidus as a target of venous approach. AJNR Am J Neuroradiol 2004;25:69-73

6. Patsalides A, Knopman J, Santillan A, Tsiouris AJ, Riina H, Gobin YP. Endovascular treatment of spinal arteriovenous lesions: beyond the dural fistula. AJNR Am J Neuroradiol 2011;32:798808

7. Jiang L, Huang CG, Liu P, Yan B, Chen JX, Chen HR, et al. 3Dimensional rotational angiography for the treatment of spinal cord vascular malformations. Surg Neurol 2008;69:369-373

8. Prestigiacomo CJ, Niimi Y, Setton A, Berenstein A. Threedimensional rotational spinal angiography in the evaluation and treatment of vascular malformations. AJNR Am J Neuroradiol 2003;24:1429-1435

9. Matsubara N, Miyachi S, Izumi T, Ohshima T, Tsurumi A, Hososhima $\mathrm{O}$, et al. Usefulness of three-dimensional digital subtraction angiography in endovascular treatment of a spinal dural arteriovenous fistula. J Neurosurg Spine 2008;8:462-467

10. Aadland TD, Thielen KR, Kaufmann TJ, Morris JM, Lanzino G, Kallmes DF, et al. 3D C-arm conebeam CT angiography as an adjunct in the precise anatomic characterization of spinal dural arteriovenous fistulas. AJNR Am J Neuroradiol 2010;31:476-480

11. Berenstein A, Lasjaunias P, ter Brugge KG. Spinal Dural Arteriovenous Fistulae. In: Berenstein A, Lasjaunias P, ter Brugge KG. Surgical Neuroangiography. Clinical and endovascular treatment aspects in adults, 2nd ed. Heidelberg: Springer, 2003:849-872

12. Berenstein A, Lasjaunias P, ter Brugge KG. Spinal and spinal cord arteries and veins. In: Berenstein A, Lasjaunias P, Ter Brugge KG. Surgical Neuroangiography. Clincal vascular anatomy and variations, 2nd ed. New York: Springer, 2001:73-164

13. Shi HB, Suh DC, Lee HK, Lim SM, Kim DH, Choi CG, et al. Preoperative transarterial embolization of spinal tumor: embolization techniques and results. AJNR Am J Neuroradiol 1999;20: 2009-2015 